\title{
Theory Analysis and Experiment Research of the Leg Mechanism for the Human-Carrying Walking Chair Robot
}

\author{
Lingfeng Sang, ${ }^{1}$ Hongbo Wang, ${ }^{1,2}$ Shuaishuai Wang, ${ }^{1}$ Na Chen, ${ }^{1}$ and Yuehua Wen ${ }^{1}$ \\ ${ }^{1}$ Parallel Robot and Mechatronic System Laboratory of Hebei Province, Yanshan University, Qinhuangdao 066004, China \\ ${ }^{2}$ Key Laboratory of Advanced Forging \& Stamping Technology and Science of Ministry of Education, \\ Yanshan University, Qinhuangdao 066004, China
}

Correspondence should be addressed to Hongbo Wang; hongbo_w@ysu.edu.cn

Received 5 May 2014; Revised 30 October 2014; Accepted 11 November 2014; Published 10 December 2014

Academic Editor: Tarek M. Sobh

Copyright (C) 2014 Lingfeng Sang et al. This is an open access article distributed under the Creative Commons Attribution License, which permits unrestricted use, distribution, and reproduction in any medium, provided the original work is properly cited.

\begin{abstract}
For the high carrying capacity of the human-carrying walking chair robot, in this paper, 2-UPS+UP parallel mechanism is selected as the leg mechanism; then kinematics, workspace, control, and experiment of the leg mechanism are researched in detail. Firstly, design of the whole mechanism is described and degrees of freedom of the leg mechanism are analyzed. Second, the forward position, inverse position, and velocity of leg mechanism are studied. Third, based on the kinematics analysis and the structural constraints, the reachable workspace of 2-UPS+UP parallel mechanism is solved, and then the optimal motion workspace is searched in the reachable workspace by choosing the condition number as the evaluation index. Fourth, according to the theory analysis of the parallel leg mechanism, its control system is designed and the compound position control strategy is studied. Finally, in optimal motion workspace, the compound position control strategy is verified by using circular track with the radius $100 \mathrm{~mm}$; the experiment results show that the leg mechanism moves smoothly and does not tremble obviously. Theory analysis and experiment research of the single leg mechanism provide a theoretical foundation for the control of the quadruped human-carrying walking chair robot.
\end{abstract}

\section{Introduction}

Walking aids have been a research hot point for several years [1]. The human-carrying walking chair robot, which is one of walking aids, could help the elderly and the lower limb disabled walk freely in the outside and navigate on uneven ground.

The human-carrying walking chair robot, which is different from the wheeled robot and the ordinary legged robot, not only needs to steadily walk by using leg mechanism as supporting point $[2,3]$, but also needs to bear weight from itself and different passengers [4]. These put forward higher requirements for performance of the leg mechanism of the walking chair robot. At present, most of humancarrying walking chair robots for the elderly and the lower limb disabled are implemented by selecting serial mechanism as leg mechanism [5-9], such as the I-Foot robot, the Hubo FX-1 robot, and the Hyperion4 robot. Using the serial mechanism as the leg mechanism, the whole volume and weight of the robot are bigger and the carrying capacity is smaller. For instance, the robot I-Foot is $200 \mathrm{~kg}$ in mass and can carry a person with $60 \mathrm{~kg}$; Hubo FX-1 is $150 \mathrm{~kg}$ in mass and can bear the load of $100 \mathrm{~kg}$. Compared with the serial mechanism, parallel mechanism (PM) can make up for the deficiencies of the serial mechanism and form the complementary relationship with the serial mechanism [1012]. For example, the WL-16RIV biped walking chair robot which was designed by Waseda University is $68 \mathrm{~kg}$ in mass and can bear a person with $75 \mathrm{~kg}$, or the goods with $80 \mathrm{~kg}$. So PM is the best choice for the leg mechanism of humancarrying walking chair robot.

Since the PM with limited DOF (degrees of freedom) has the advantages such as the simple mechanical structure and low cost in design, manufacturing, and control, the PM with less than 6 DOF is widely used. Analyzing the leg mechanism of the human-carrying walking chair robot, we could find that its DOF are 3 (including swing back and forth, swing left and right, and lifting up and down). Configurations of meeting 
motion requirement are symmetrical 3-UPU PM [13], 3-PUU PM [14], and 3-RPS PM [15] and asymmetrical 2-UPS+UP $\mathrm{PM}, \mathrm{UPS}+\mathrm{SPR}+\mathrm{SP} \mathrm{PM}$, and UPS+UP+UPR PM. The 3-UPU $\mathrm{PM}$ is difficult to ensure that the axis of two universal joints for each branch is parallel when the installation is finished. The arrangement of the prismatic joint for 3-PUU PM is difficult and the 3-PUU PM has poor motion performance as the leg mechanism. Each branch of the 3-RPS PM has a constraint force on the moving platform. If 3-RPS PM is selected as the leg mechanism of the walking chair robot, the body mechanism of the walking chair robot may not move because of the constraint forces coupling of the multiple branches. Each branch of asymmetrical UPS+SPR+SP PM and UPS+UP+UPR PM is different, so it is difficult to modular design and manufacturing each branch of the leg mechanism. The motion of asymmetrical 2-UPS+UP PM is determined by the UP branch, and two branches are the same in the whole leg mechanism. So it is easy to be designed and manufactured.

The output workspace of the reference point in the moving platform of the PM is one of the important indexes for PM performance. In general, the numerical method and geometry method are used to solve PM workspace. The numerical method, namely, the reachable workspace of the leg mechanism, is obtained by analyzing the constraint relationship and inverse position kinematics of the mechanism [16-19]. The geometry method, namely, the reachable workspace of the leg mechanism, is solved by analyzing constraint relationship of the leg mechanism and using computer software [20-22]. However, the reachable workspace conducted by these two methods cannot be applied to the control of the PM because of existing poor performance areas, so the optimal motion workspace needs to be found in reachable workspace further [23].

In this paper, the 2-UPS+UP parallel leg mechanism (PLM) of the human-carrying walking chair robot is discussed in detail. First of all, according to the prototype model, the forward position kinematics, inverse position kinematics, and inverse velocity kinematics are analyzed. Based on the design size and the structural constraints, the reachable workspace of the 2-UPS+UP PLM is solved. Selecting the condition number as the evaluation index, the optimal workspace is found in the reachable workspace and this workspace is used as the motion workspace. Then, on basis of the motion workspace and kinematics analysis, the control system of 2-UPS+UP PLM is designed and the compound position control strategy is studied. Finally, in optimal workspace, the compound position control strategy is verified by using circular trajectory with the radius $100 \mathrm{~mm}$.

\section{Design of the Whole Mechanism}

The prototype of the human-carrying walking chair robot is shown in Figure 1. The whole mechanism consists of seat, upper platform, leg mechanism, and control system; among them, the seat, upper platform, and control system are collectively called the body mechanism. The photo and

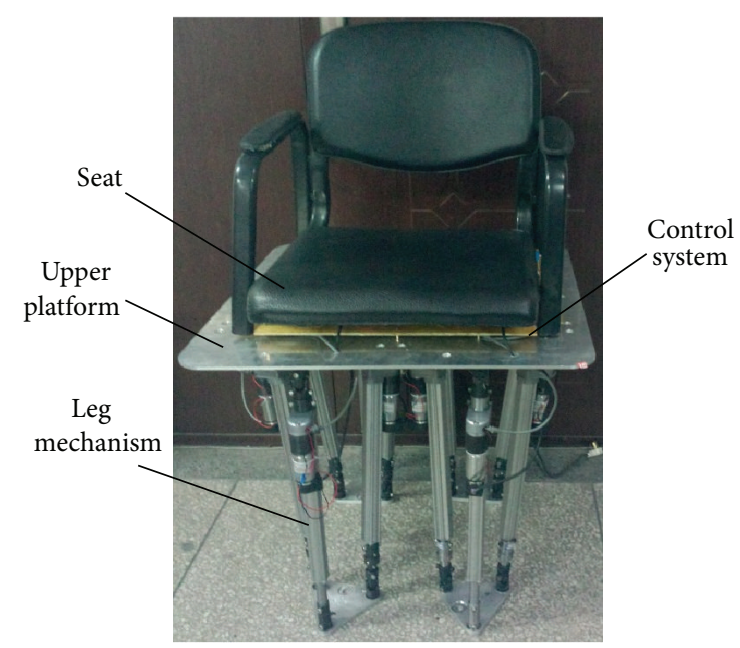

FIGURE 1: Human-carrying walking chair robot.

the structure sketch of the single leg mechanism which is 2UPS+UP PM are shown in Figures 2 and 3.

This leg mechanism consists of the upper platform $A$, the lower platform $E$, two UPS branches, and one UP branch. The $S$ joint of the UPS branch is made up of one rotation joint and one universal joint, as shown in Figure 2 and the points $U_{5}, U_{6}$ in Figure 3 . Both of the upper and lower platforms are equilateral triangles and their sides are $a$ and $b$, respectively. Consider $\angle A_{2} A_{1} A_{3}=2 \Phi, \angle E_{2} E_{1} E_{3}=2 \varphi$. The hinge points among three branches and the upper platform and the lower platform are $A_{i}, E_{i}(i=1,2,3)$, respectively. The global coordinate frame $A_{1}-X Y Z$ is established at point $A_{1}$ on the upper platform. Axis $X$ is parallel to $A_{2} A_{3}$. Axis $Z$ is upward and vertical to the upper platform, and axis $Y$ is determined by the right-hand screw rule. The moving coordinate frame $E_{1}-X_{E} Y_{E} Z_{E}$ is fixed at point $E_{1}$. Axis $X_{E}$ is parallel to $E_{2} E_{3}$. Axis $Z_{E}$ is upward and vertical to the lower platform, and axis $Y_{E}$ is determined by the right-hand screw rule. According to Figure 2, some structural parameters are defined as follows. $t_{1}, t_{2}$ are the thickness of the upper platform and the lower platform. $d_{1}$ is the distance between the connecting hole of connector 1 and the end face of the upper platform. $d_{2}$ is the distance between the center of universal joint and the connecting hole of universal joint. $d_{3}$ is the distance between the two connecting holes of connector $2 . d_{4}$ is the distance between the two connecting holes of connector $3 . d_{5}$ is the distance between the connecting hole of connector 4 and the end face of the lower platform. $d_{6}$ is the length of the rotation joint. The design value of these parameters is shown in Table 1.

According to $G-K$ formula [24], the DOF of the 2UPS+UP PM could be obtained by

$$
\begin{aligned}
M & =d(n-g-1)+\sum_{i=1}^{g} f_{i}+v \\
& =6(7-8-1)+15+0=3,
\end{aligned}
$$

where $d$ is the order number of mechanism, $n$ is the number of mechanism components, $g$ is the number of kinematic joints, 


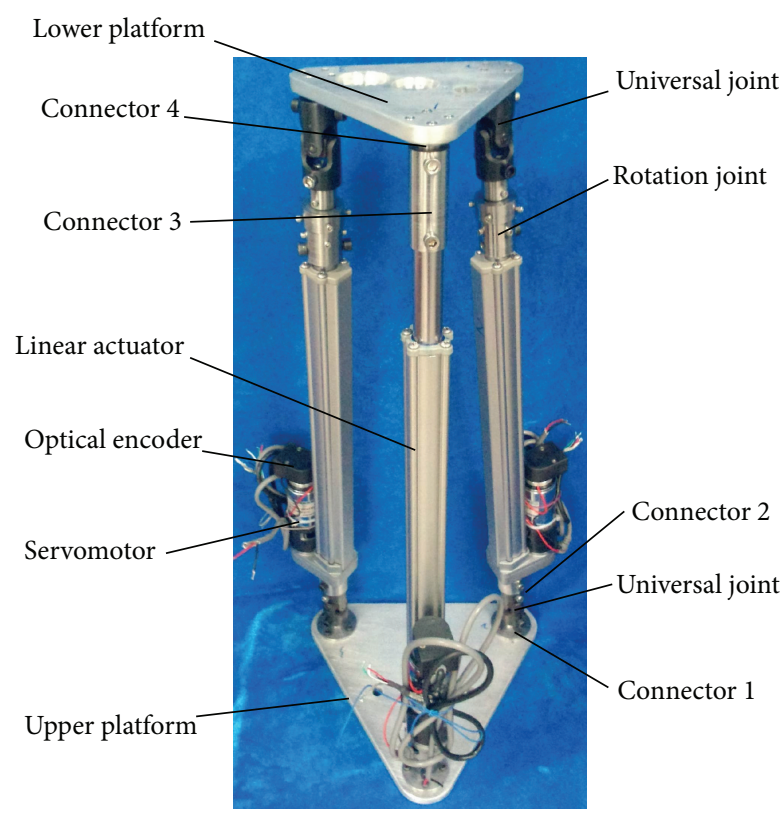

FIgURE 2: 2-UPS+UP PLM.

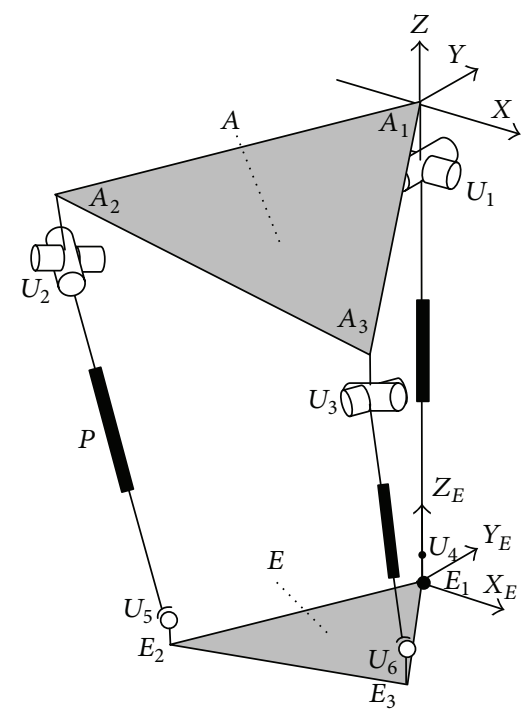

FIGURE 3: Structure sketch of 2-UPS+UP PLM.

$f_{i}$ is the number of DOF for the $i$ th kinematics joint, and $v$ is the number of the redundant constraints of the mechanism.

Based on (1), we find that the single 2-UPS+UP PM which is elected as the leg mechanism cannot meet the motion requirements of the whole mechanism, so the passive sphere is added in the end face of the lower platform.

In order to ensure contact force of the end point of the leg mechanism along the direction of the branch, the sphere joint is added in the junction between each branch of the leg mechanism and the lower platform, as shown in Figure 4. At this point, the leg mechanism consists of 2-UPS+UP PLM and foot. For the foot section, there are three motion states: one sphere joint contacting with the ground, two sphere joints contacting with the ground, and three sphere
TABLE 1: Design values of the mechanism parameters.

\begin{tabular}{lccc}
\hline Parameter & Value & Parameter & Value \\
\hline$a$ & $200 \mathrm{~mm}$ & $b$ & $120 \mathrm{~mm}$ \\
$\Phi$ & $\pi / 6$ & $\varphi$ & $\pi / 6$ \\
$t_{1}$ & $10 \mathrm{~mm}$ & $t_{2}$ & $10 \mathrm{~mm}$ \\
$d_{1}$ & $21 \mathrm{~mm}$ & $d_{2}$ & $23 \mathrm{~mm}$ \\
$d_{3}$ & $25 \mathrm{~mm}$ & $d_{4}$ & $44 \mathrm{~mm}$ \\
$d_{5}$ & $20 \mathrm{~mm}$ & $d_{6}$ & $62 \mathrm{~mm}$ \\
\hline
\end{tabular}

joints contacting with the ground. The DOF of each state are analyzed as follows.

(1) One Sphere Joint Contacting with the Ground. Under this state, the DOF of the foot section is computed as

$$
\begin{aligned}
M & =d(n-g-1)+\sum_{i=1}^{g} f_{i}+v \\
& =6(2-1-1)+3+0=3 .
\end{aligned}
$$

(2) Two Sphere Joints Contacting with the Ground. Under this state, the DOF of the foot section are obtained as

$$
\begin{aligned}
M & =d(n-g-1)+\sum_{i=1}^{g} f_{i}+v \\
& =6(2-2-1)+6+3=3 .
\end{aligned}
$$

(3) Three Sphere Joints Contacting with the Ground. Under this state, the DOF of the foot section are solved as

$$
\begin{aligned}
M & =d(n-g-1)+\sum_{i=1}^{g} f_{i}+v \\
& =6(2-3-1)+9+6=3 .
\end{aligned}
$$

It is seen by (2)-(4) that the foot section of the single leg mechanism could be simplified as a sphere joint with $3 \mathrm{DOF}$. So the DOF of the whole leg mechanism could be computed as

$$
\begin{aligned}
M & =d(n-g-1)+\sum_{i=1}^{g} f_{i}+v \\
& =6(8-9-1)+18+0=6 .
\end{aligned}
$$

The DOF of the single leg mechanism is 6; that is to say, the leg mechanism has no constraint to the body mechanism. So the DOF of the body mechanism is 6 .

Due to the fact that the motion of the foot section has no effect on the leg mechanism motion, only 2-UPS+UP PLM mechanism is analyzed.

\section{Position Analysis of the Leg Mechanism}

Position analysis of the leg mechanism is very important to the kinematics analysis and the control of the mechanism. So the forward and inverse position analysis is conducted in this section. 


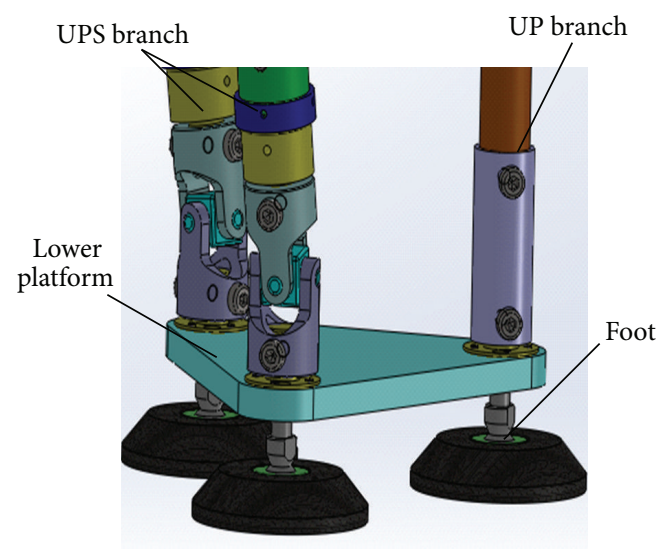

FIGURE 4: 2-UPS+UP PM and foot.

3.1. Inverse Position Analysis. Inverse position analysis is to find length change of each branch when the position of the point $E_{1}$ is known.

According to the coordinate frame established in Figure 3, each point of the upper platform in the global coordinate frame could be described as

$$
\mathbf{A}_{1}=\left[\begin{array}{l}
0 \\
0 \\
0
\end{array}\right], \quad \mathbf{A}_{2}=\left[\begin{array}{c}
-a s \phi \\
-a c \phi \\
0
\end{array}\right], \quad \mathbf{A}_{3}=\left[\begin{array}{c}
a s \phi \\
-a c \phi \\
0
\end{array}\right],
$$

where $s \phi=\sin (\phi), c \phi=\cos (\phi)$.

The center point of the upper universal joint in the global coordinate frame could be written as

$$
\mathbf{U}_{11}=\left[\begin{array}{c}
0 \\
0 \\
-x_{1}
\end{array}\right], \quad \mathbf{U}_{12}=\left[\begin{array}{c}
-a s \phi \\
-a c \phi \\
-x_{1}
\end{array}\right], \quad \mathbf{U}_{13}=\left[\begin{array}{c}
a s \phi \\
-a c \phi \\
-x_{1}
\end{array}\right] \text {, }
$$

where $x_{1}$ is the design size of the leg mechanism and $x_{1}=$ $t_{1}+d_{1}+d_{2}$.

Each point of the lower platform in the moving coordinate frame could be described as

$$
\mathbf{E}_{1}^{\prime}=\left[\begin{array}{l}
0 \\
0 \\
0
\end{array}\right], \quad \mathbf{E}_{2}^{\prime}=\left[\begin{array}{c}
-b s \varphi \\
-b c \varphi \\
0
\end{array}\right], \quad \mathbf{E}_{3}^{\prime}=\left[\begin{array}{c}
b s \varphi \\
-b c \varphi \\
0
\end{array}\right] .
$$

The lower endpoint of the UP branch and the center point of the lower universal joint in the UPS branch could be expressed as

$$
\mathbf{U}_{14}^{\prime}=\left[\begin{array}{c}
0 \\
0 \\
x_{2}
\end{array}\right], \quad \mathbf{U}_{15}^{\prime}=\left[\begin{array}{c}
-b s \varphi \\
-b c \varphi \\
x_{3}
\end{array}\right], \quad \mathbf{U}_{16}^{\prime}=\left[\begin{array}{c}
b s \varphi \\
-b c \varphi \\
x_{3}
\end{array}\right],
$$

where $x_{2}, x_{3}$ are the design size of the leg mechanism and $x_{2}=d_{5}, x_{3}=d_{2}+d_{5}$.

Each point in the moving coordinate frame could be transformed to the global coordinate frame as follows:

$$
\mathbf{U}_{1 k}={ }_{E_{1}}^{A} \mathbf{R} \mathbf{U}_{1 k}^{\prime}+\mathbf{E}_{1}, \quad(k=4,5,6),
$$

where ${ }_{E_{1}}^{A} \mathbf{R}$ is the rotation transformation matrix from the moving coordinate frame to the global coordinate frame and ${ }_{E_{1}}^{A} \mathbf{R}=\left[\begin{array}{lll}x_{l} & y_{l} & z_{l} \\ x_{m} & y_{m} & z_{m} \\ x_{n} & y_{n} & z_{n}\end{array}\right] . \mathbf{E}_{1}$ is the position vector of point $E_{1}$, and $\mathbf{E}_{1}=\left[\begin{array}{lll}X_{E} & Y_{E} & Z_{E}\end{array}\right]^{\mathrm{T}}$.

Due to the constraint of the UP branch, the geometric constraint relationship of the leg mechanism can be written as follows:

$$
\mathbf{R}_{1} \perp \mathbf{R}_{2}, \quad \mathbf{R}_{\mathbf{2}} \perp \mathbf{n}_{11}, \quad \mathbf{n}_{11} / / \mathbf{Z}_{E}, \quad \mathbf{R}_{2} / / \mathbf{X}_{E},
$$

where $\mathbf{R}_{1}$ and $\mathbf{R}_{2}$ are the first and second revolute axes of the universal joint $U$ of branch $U P$, respectively; $\mathbf{n}_{11}$ is the direction vector of the UP branch.

According to the established coordinate frame, the following equation can be obtained:

$$
\mathbf{R}_{1}=\left[\begin{array}{l}
0 \\
1 \\
0
\end{array}\right], \quad \mathbf{R}_{2}=\left[\begin{array}{c}
x_{l} \\
x_{m} \\
x_{n}
\end{array}\right], \quad \mathbf{n}_{11}=\frac{1}{l_{11}}\left(\mathbf{U}_{14}-\mathbf{U}_{11}\right)
$$

Putting (9), (10), and (12) into (11), the following equation can be obtained:

$$
\begin{gathered}
x_{m}=0, \\
X_{E}=-\left(l_{11}+x_{2}\right) z_{l}, \\
Y_{E}=-\left(l_{11}+x_{2}\right) z_{m}, \\
Z_{E}=-\left(l_{11}+x_{2}\right) z_{n}-x_{1} .
\end{gathered}
$$

According to (13), $Y-X-Z$ Euler angles are selected, and the final transformation matrix can be expressed as follows:

$$
{ }_{E_{1}}^{A} \mathbf{R}=\left[\begin{array}{ccc}
c \alpha & s \alpha s \beta & s \alpha c \beta \\
0 & c \beta & -s \beta \\
-s \alpha & c \alpha s \beta & c \alpha c \beta
\end{array}\right]
$$

where $\alpha$ and $\beta$ represent the rotation angle along axis $Y$ and axis $X$ in the global coordinate frame.

Combining (13) with (14), the following expressions are obtained:

$$
\begin{gathered}
l_{11}=\sqrt{X_{E}^{2}+Y_{E}^{2}+\left(Z_{E}+x_{1}\right)^{2}}-x_{2}, \\
\alpha=\arctan \left(\frac{X_{E}}{x_{1}+Z_{E}}\right), \\
\beta=\arcsin \left(\frac{Y_{E}}{l_{11}+x_{2}}\right) .
\end{gathered}
$$

The length change of each branch could be expressed as

$$
\mathbf{l}_{1 i}=\mathbf{U}_{1 k}-\mathbf{U}_{1 i}
$$


According to (16), the final expressions for the length change of UPS branch could be derived as

$$
\begin{aligned}
l_{12}= & \left(-b s \varphi c \alpha-b c \varphi s \alpha s \beta+x_{3} s \alpha c \beta+X_{E}+a s \phi\right)^{2} \\
& +\left(-b c \varphi c \beta-x_{3} s \beta+Y_{E}+a c \phi\right)^{2} \\
& \left.+\left(b s \varphi s \alpha-b c \varphi c \alpha s \beta+x_{3} c \alpha c \beta+Z_{E}+x_{1}\right)^{2}\right)^{1 / 2}, \\
l_{13}= & \left(\left(b s \varphi c \alpha-b c \varphi s \alpha s \beta+x_{3} s \alpha c \beta+X_{E}-a s \phi\right)^{2}\right. \\
& +\left(-b c \varphi c \beta-x_{3} s \beta+Y_{E}+a c \phi\right)^{2} \\
& \left.+\left(-b s \varphi s \alpha-b c \varphi c \alpha s \beta+x_{3} c \alpha c \beta+Z_{E}+x_{1}\right)^{2}\right)^{1 / 2} .
\end{aligned}
$$

3.2. Direct Position Analysis. Forward position analysis is to find the position of the point $E_{1}$ when the length change of each branch is given.

The two equations of (17) can be simplified by squaring then adding and subtracting as follows:

$$
\begin{gathered}
c \alpha=\frac{t_{4}-4 a b c \phi c \varphi c \beta-4 a t_{3} c \phi s \beta}{4 a b s \phi s \varphi}, \\
s \alpha=\frac{t_{5}}{4 a s \phi\left(t_{3} c \beta-b c \varphi s \beta\right)},
\end{gathered}
$$

where $t_{3}=x_{3}-l_{11}-x_{2}, t_{4}=2 t_{3}^{2}+2 a^{2}+2 b^{2}-l_{12}^{2}-l_{13}^{2}$, and $t_{5}=l_{12}^{2}-l_{13}^{2}$.

Observing (18), we could find that only $\alpha, \beta$ are unknown. To solve (18), let $u=\tan (\alpha / 2), v=\tan (\beta / 2)$.

Putting trigonometric functions $s \alpha=2 u /\left(1+u^{2}\right), c \alpha=$ $\left(1-u^{2}\right) /\left(1+u^{2}\right), s \beta=2 v /\left(1+v^{2}\right)$, and $c \beta=\left(1-v^{2}\right) /\left(1+v^{2}\right)$ into (18), we have

$$
\begin{gathered}
k_{12} u^{2}+k_{10}=0, \\
k_{22} u^{2}+k_{21} u+k_{20}=0,
\end{gathered}
$$

where

$$
\begin{aligned}
k_{12}= & \left(t_{4}+4 a b c \phi c \varphi+4 a b s \phi s \varphi\right) v^{2}-8 a t_{3} c \phi v+t_{4} \\
& -4 a b c \phi c \varphi+4 a b s \phi s \varphi, \\
k_{10}= & \left(t_{4}+4 a b c \phi c \varphi-4 a b s \phi s \varphi\right) v^{2}-8 a t_{3} c \phi v+t_{4} \\
& -4 a b c \phi c \varphi-4 a b s \phi s \varphi,
\end{aligned}
$$

and

$$
\begin{gathered}
k_{22}=t_{5} v^{2}+t_{5}, \\
k_{21}=8 a t_{3} s \phi v^{2}+16 a b s \phi c \varphi v-8 a t_{3} s \phi, \\
k_{20}=t_{5} v^{2}+t_{5} .
\end{gathered}
$$

Multiplying both sides by $u$ in (19), the other two equations could be obtained. These four equations can be transformed into matrix form as follows:

$$
\mathbf{A U}=0 \text {, }
$$

where

$$
\mathbf{A}=\left[\begin{array}{cccc}
k_{12} & 0 & k_{10} & 0 \\
0 & k_{12} & 0 & k_{10} \\
k_{22} & k_{21} & k_{20} & 0 \\
0 & k_{22} & k_{21} & k_{20}
\end{array}\right], \quad \mathbf{U}=\left[\begin{array}{c}
u^{3} \\
u^{2} \\
u \\
1
\end{array}\right]
$$

A is a matrix about the variable $u$. Because of $\mathbf{U} \neq 0$, so the sufficient and necessary condition of (22) with nonzero solution is that the determinant of coefficient matrix $A$ is equal to zero. The equation of the variable $v$ can be obtained from the matrix $\mathbf{A}$ as follows:

$$
k_{12}^{2} k_{20}^{2}+k_{10}^{2} k_{22}^{2}+k_{12} k_{10} k_{21}^{2}-2 k_{10} k_{12} k_{22} k_{20}=0 .
$$

Observing (24), it can be found that the highest power of the variable $v$ is 8 . That is to say, there are 8 real roots at most in (24). After solving the variable $v$, the variable $u$ could be computed by the following equation:

$$
u=\frac{k_{10} k_{22}-k_{12} k_{20}}{k_{12} k_{21}} .
$$

On basis of the above solving, value of the variables $\alpha, \beta$ could be computed; then the value of the point $\mathbf{E}_{1}$ could be obtained.

\section{Velocity Analysis of the Leg Mechanism}

According to (16), the length change of each branch in the leg mechanism can be rewritten as

$$
\begin{gathered}
\mathbf{l}_{11}=l_{11} \mathbf{n}_{11}, \\
\mathbf{l}_{11}+\mathbf{b}_{45}=\mathbf{a}_{12}+\mathbf{l}_{12}, \\
\mathbf{l}_{11}+\mathbf{b}_{46}=\mathbf{a}_{13}+\mathbf{l}_{13},
\end{gathered}
$$

where $\mathbf{l}_{1 i}$ is the length change of each branch, $\mathbf{b}_{45}=\mathbf{U}_{15}-\mathbf{U}_{14}$, $\mathbf{a}_{12}=\mathbf{U}_{12}-\mathbf{U}_{11}, \mathbf{b}_{46}=\mathbf{U}_{16}-\mathbf{U}_{14}$, and $\mathbf{a}_{13}=\mathbf{U}_{13}-\mathbf{U}_{11}$.

Taking one derivative to both sides of each equation in (26), the velocity of each branch can be got:

$$
\begin{gathered}
\mathbf{v}_{11}=i_{11} \mathbf{n}_{11}+\mathbf{w}_{11} \times l_{11} \mathbf{n}_{11}, \\
\mathbf{v}_{11}=\dot{l}_{12} \mathbf{n}_{12}+\mathbf{w}_{12} \times l_{12} \mathbf{n}_{12}-\mathbf{w}_{11} \times \mathbf{b}_{45}, \\
\mathbf{v}_{11}=\dot{l}_{13} \mathbf{n}_{13}+\mathbf{w}_{13} \times l_{13} \mathbf{n}_{13}-\mathbf{w}_{11} \times \mathbf{b}_{46},
\end{gathered}
$$

where $\mathbf{n}_{1 i}$ is the direction vector of each branch, $i_{1 i}$ is the velocity along the branch direction of each branch, and $\mathbf{w}_{11}$ is the angular velocity of each branch.

Taking the dot product $\mathbf{n}_{1 i}$ on both sides of each equation in (27), the following equation could be derived:

$$
\dot{\mathbf{q}}_{1 i}=\left[\begin{array}{cc}
\mathbf{n}_{11}^{\mathrm{T}} & 0 \\
\mathbf{n}_{12}^{\mathrm{T}} & \left(\mathbf{b}_{45} \times \mathbf{n}_{12}\right)^{\mathrm{T}} \\
\mathbf{n}_{13}^{\mathrm{T}} & \left(\mathbf{b}_{46} \times \mathbf{n}_{13}\right)^{\mathrm{T}}
\end{array}\right]\left[\begin{array}{c}
\mathbf{v}_{11} \\
\mathbf{w}_{11}
\end{array}\right],
$$

where $\dot{\mathbf{q}}_{1 i}=\left[\begin{array}{lll}\dot{l}_{11} & \dot{l}_{12} & \dot{l}_{13}\end{array}\right]^{\mathrm{T}}$ 
Meanwhile, the velocity of the end point $\mathbf{E}_{1}$ could be expressed as

$$
\mathbf{v}_{1}=\mathbf{v}_{11}+\mathbf{w}_{11} \times \mathbf{b}_{14},
$$

where $\mathbf{b}_{14}=\mathbf{E}_{1}-\mathbf{U}_{14}$.

Taking the cross product on both sides of the first equation in (27), the relationship between the angular velocity $\mathbf{w}_{11}$ and the velocity of the point $U_{14}$ of the UP branch can be obtained:

$$
\mathbf{w}_{11}=\frac{\mathbf{n}_{11} \times \mathbf{v}_{11}}{l_{11}} .
$$

Combing (28) and (30), the following equation could be derived:

$$
\dot{\mathbf{q}}_{1 i}=\mathbf{J}_{11} \mathbf{v}_{11},
$$

where

$$
\mathbf{J}_{11}=\left[\begin{array}{c}
\mathbf{n}_{11}^{\mathrm{T}} \\
\left(\mathbf{n}_{12}+\frac{\left(\mathbf{b}_{45} \times \mathbf{n}_{12}\right) \times \mathbf{n}_{11}}{l_{11}}\right)^{\mathrm{T}} \\
\left(\mathbf{n}_{13}+\frac{\left(\mathbf{b}_{46} \times \mathbf{n}_{13}\right) \times \mathbf{n}_{11}}{l_{11}}\right)^{\mathrm{T}}
\end{array}\right] .
$$

In the same way, (29) could be transformed as

$$
\mathbf{v}_{1}=\mathbf{J}_{12} \mathbf{v}_{11},
$$

where $\mathbf{J}_{12}=\mathbf{I}-\left(\left(S\left(\mathbf{b}_{14}\right) S\left(\mathbf{n}_{11}\right)\right) / l_{11}\right)$ and $\mathbf{S}(*)$ is antisymmetric matrix.

If $\mathbf{J}_{12}$ exists the inverse matrix, transitive relation between the input and output for velocity of the leg mechanism could be expressed by the following equation:

$$
\mathbf{v}_{1}=\mathbf{J}_{12} \mathbf{v}_{11} \text {, }
$$

where $\mathbf{J}_{1}=\mathbf{J}_{11} \mathbf{J}_{12}{ }^{-1}$.

\section{Workspace Analysis}

5.1. Reachable Workspace Analysis of Leg Mechanism Workspace. The size and shape of the reachable workspace of 2-UPS+UP PM are restricted by the following factors: (1) limitation of universal joint; (2) limitation of the branch length; (3) interference among the branches. The restraint relationships could be expressed as follows:

$$
\begin{gathered}
\theta_{u i} \leq \theta_{u i \max }, \\
l_{1 i \min } \leq l_{1 i} \leq l_{1 i \max }, \\
d \leq d_{1 i},
\end{gathered}
$$

where $\theta_{u i}$ is real angle of the universal joint; $\theta_{u i \max }$ is the allowable maximal angle of the universal joint; $l_{1 i \min }$ is the minimum of the branch length; $l_{1 i \max }$ is the maximum of the branch length; $d_{1 i}$ is the shortest distance among the branches; $d$ is the maximum diameter of the upper and the lower rods of the branch.
In order to simplify the calculation, the lengths of $d_{2}, d_{3}$, $d_{4}$, and $d_{5}$ are recorded into the length of UP branch, and $d_{2}, d_{3}$, and $d_{6}$ are recorded into the length of UPS branch. According to value of design parameters and position analysis of parallel mechanism, the reachable workspace of the point $E_{1}$ could be obtained by giving the initial sphere workspace with radius $1200 \mathrm{~mm}$ and the restraint relationships of in (35). The result is shown in Figure 5. From Figure 5, it can be seen that this workspace has the following characteristics.

(1) The reachable workspace of the point $E_{1}$ is composed of inner and outer surfaces. The inner surface is the inner constraints surface of the mechanism; the outer surface is the outer constraints surface of the mechanism.

(2) The reachable workspace of the point $E_{1}$ is symmetrical about axis $x$, but it is asymmetrical about axis $y$.

5.2. Optimal Motion Workspace. Observing the reachable workspace of 2-UPS+UP PM, it could meet the workspace requirement of the leg mechanism, but some areas in the reachable workspace are not the good motion area because of the motion performance of the mechanism being poor. So the optimal motion workspace in the reachable workspace needs to be found to apply to the trajectory planning of the end point $E_{1}$.

For motion performance of mechanism, the condition number of Jacobin matrix can be used as evaluation index:

$$
\operatorname{cond}\left(\mathbf{J}_{1}\right)=\frac{\sigma_{\max }}{\sigma_{\text {min }}}=\sqrt{\frac{\lambda_{\text {max }}\left(\mathbf{J}_{1}^{\mathrm{T}} \mathbf{J}_{1}\right)}{\lambda_{\text {min }}\left(\mathbf{J}_{1}^{\mathrm{T}} \mathbf{J}_{1}\right)}},
$$

where $\operatorname{cond}\left(\mathbf{J}_{1}\right)$ is the condition number of Jacobin matrix, $\sigma_{\max }$ is the largest singular value, $\sigma_{\min }$ is the smallest singular value, $\lambda_{\max }$ is the maximum eigenvalue of the matrix $\mathbf{J}_{1}^{\mathrm{T}} \mathbf{J}_{1}$, and $\lambda_{\min }$ is the minimum eigenvalue of the matrix $\mathbf{J}_{1}^{\mathrm{T}} \mathbf{J}_{1}$.

The condition number indicates the distortion of input and output of the mechanism. If the value of the condition number is smaller, the motion performance of the mechanism is better. So the change of the condition number in the whole reachable workspace needs to be analyzed.

Using simulation software, the change of the condition number in the whole reachable workspace can be obtained as shown in Figure 6. Observing these figures, the following conclusions could be obtained.

(1) With the increase of height, the condition number of the leg mechanism is gradually decreased.

(2) At the same height, with the increase of the values $x, y$, the condition number of the leg mechanism increases gradually and increase to the maximum when reaching the edge of the reachable workspace. Hence the leg mechanism should avoid movement in the reachable workspace boundary as soon as possible when the trajectory planning.

(3) Starting from the height $-700 \mathrm{~mm}$, the change of the condition number is stable. 


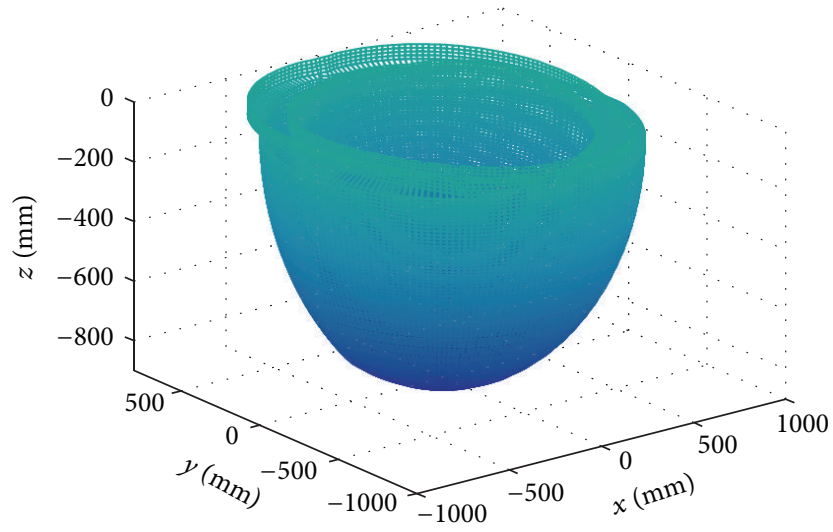

(a) Three-dimensional workspace surface

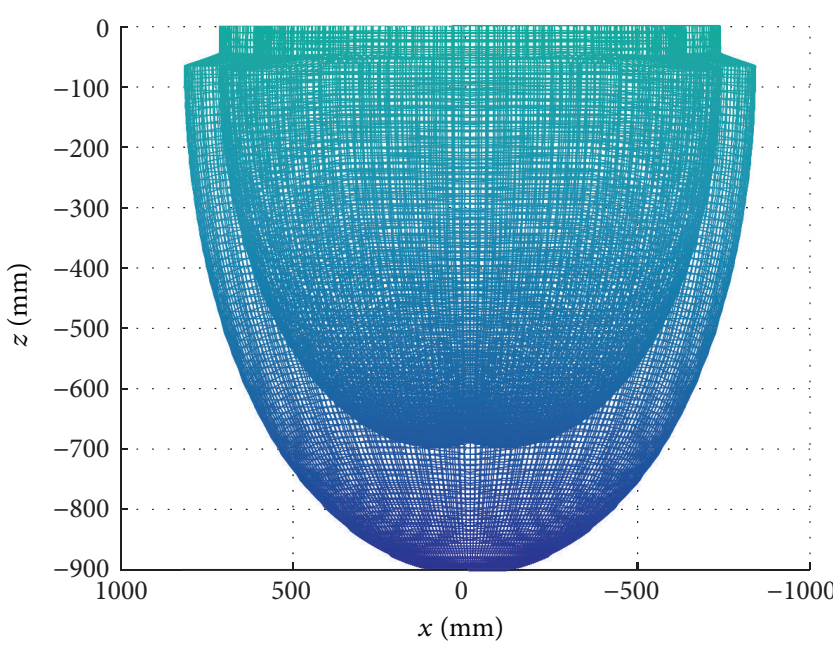

(b) Projection of workspace in $x o z$ plane

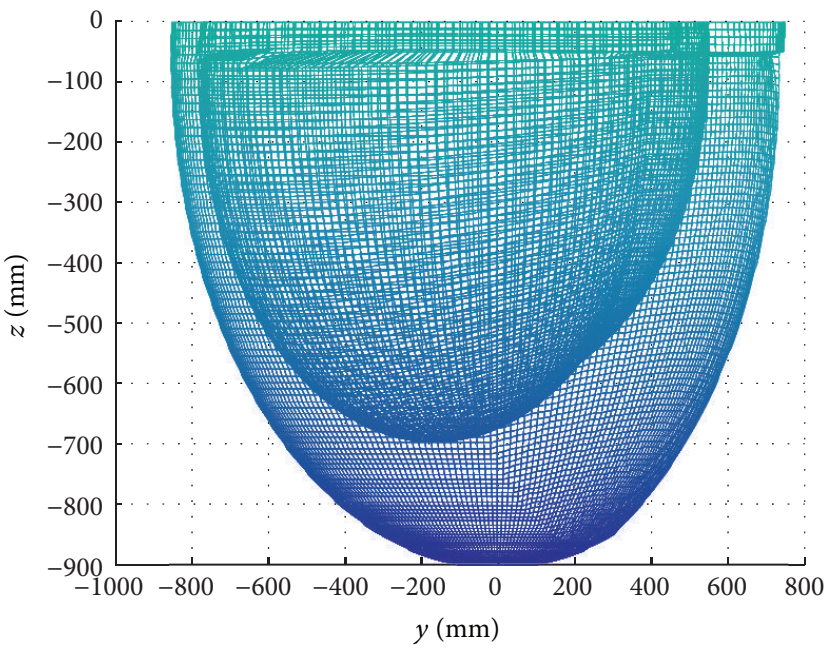

(c) Projection of workspace in yoz plane

FIgURE 5: The reachable workspace of 2-UPS+UP PM.

(4) Seeing Figures 6(d), 6(e), and 6(f), it can be found that the value and fluctuate of the condition number for the leg mechanism are smaller when the reachable workspace is in the ranges $-200 \leqslant x \leqslant 200 \mathrm{~mm}$, $-300 \leqslant y \leqslant 300 \mathrm{~mm}$, and $-850 \leqslant z \leqslant-650 \mathrm{~mm}$. So this area can be chosen as the optimal motion workspace of the leg mechanism.

\section{Experiment Research of the Leg Mechanism}

Experiment research of the leg mechanism is very important to the whole human-carrying walking chair robot. In order to build the control system of the walking chair robot, the motor controller and motor driver are developed by our research group as shown in Figure 7. The single chip computer PIC18F452 is used for motor controller, and CPLD chip XC9536-7VQ44C is applied for motor driver. This controller could realize three control modes (position control, velocity control, and current control), and the three control modes could be switched at any time.

The whole hardware system consists of the leg mechanism, the control system, power modular, and host computer as shown in Figure 8. The control system includes three controllers and three drivers to control servomotor of the three branches, respectively. There are two power modules. One power module converts voltage from AC $220 \mathrm{v}$ to DC $24 \mathrm{v}$ to supply the driver energy, and the other converts from DC 24 to DC $9 \mathrm{v}$ to supply the controller energy. Communication way of the controller is RS-485, while the host computer is RS-232; so serial port conversion module is added. Using LabView software, control program is written in host computer.

6.1. Kinematics Control Strategy Based on the Joint Space. The control strategy based on the joint space is that every branch of the PLM is regarded as single input single output system. That is to say, the position and orientation of the moving platform could be realized by controlling the motion of each branch. The control block diagram is shown in Figure 9. The control strategy is described as follows.

First of all, the motion trajectory for the reference point of the moving platform is planned in the optimal workspace. Second, according to control cycle, the planning motion 


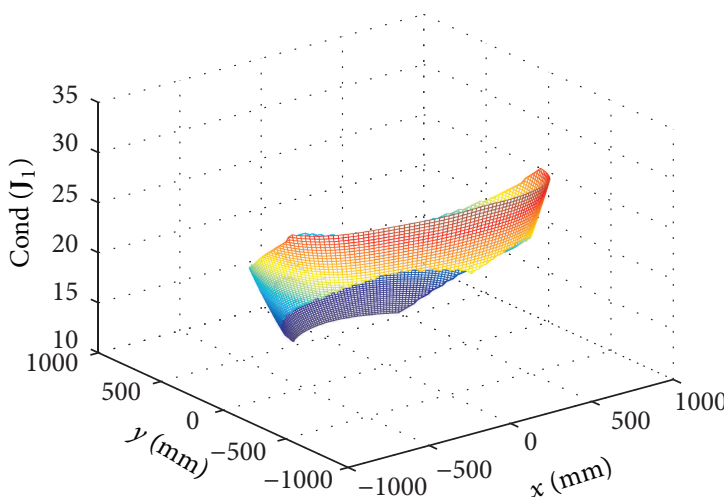

(a) $z=-400 \mathrm{~mm}$

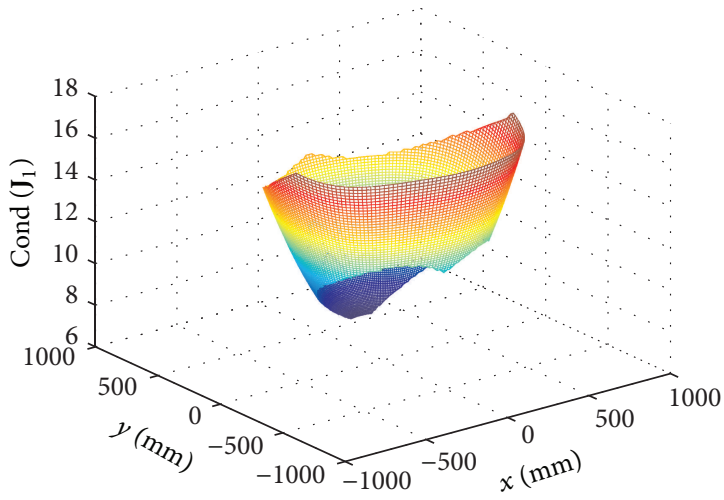

(c) $z=-600 \mathrm{~mm}$

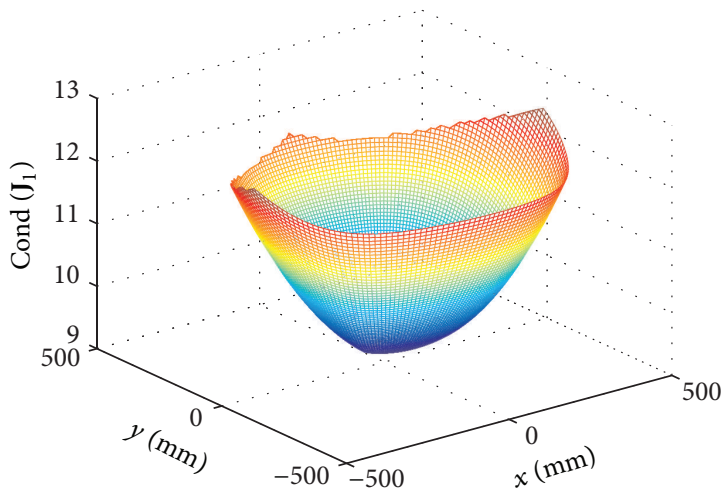

(e) $z=-800 \mathrm{~mm}$

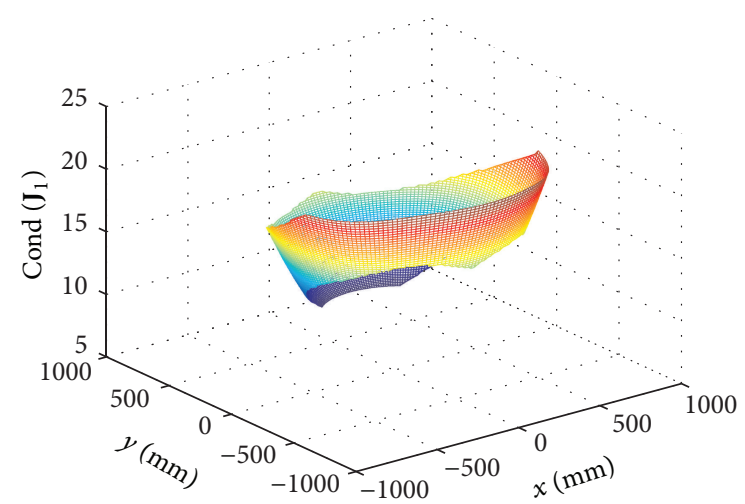

(b) $z=-500 \mathrm{~mm}$

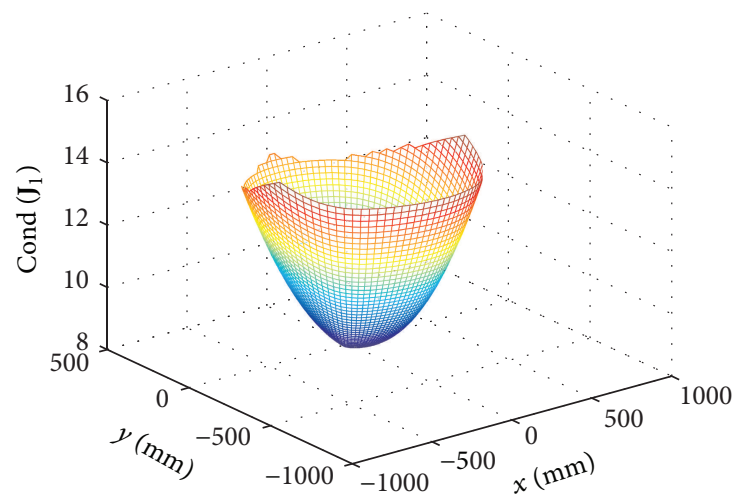

(d) $z=-700 \mathrm{~mm}$

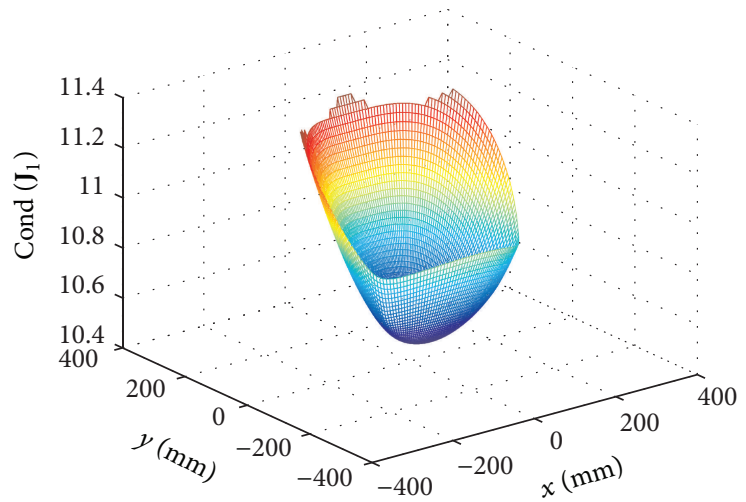

(f) $z=-900 \mathrm{~mm}$

FIGURE 6: Changes of Jacobian matrix of the condition number in the reachable workspace.

trajectory of the moving platform is discretized. Method of the discretization is as follows.

(I) Based on the planning trajectory for the reference point of the moving platform and the inverse kinematics of the leg mechanism, the motion planning trajectory of each branch is computed by MATLAB software.

(II) The initial position for the motion planning trajectory of each branch is offset to 0 , and then the motion control trajectory could be obtained.
(III) Based on control cycle, the motion control trajectory of each branch is discretized and the desired position and velocity for instruction of the controller is obtained.

Finally, according to control cycle, the instruction is set to the corresponding controller and the motion planning trajectory of each branch is realized.

In the motion process, the controller uses the compound position control strategy to control the motion of the leg mechanism. The compound position control strategy is that the mechanism is controlled by constantly switching the position control mode and the velocity control mode. The 


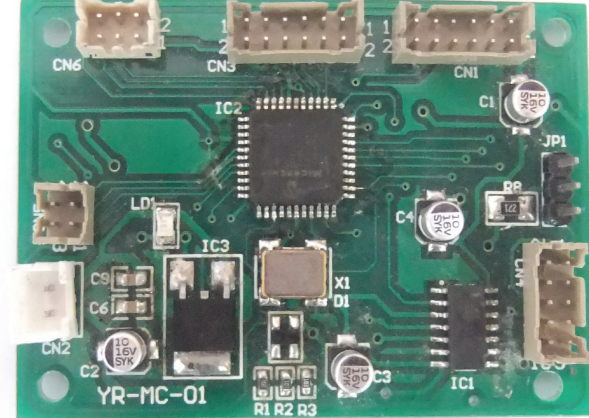

(a) Motor controller

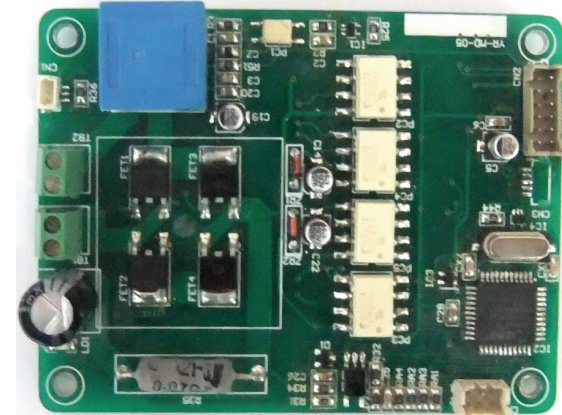

(b) Motor driver

FIGURE 7: The controller and driver of motor.

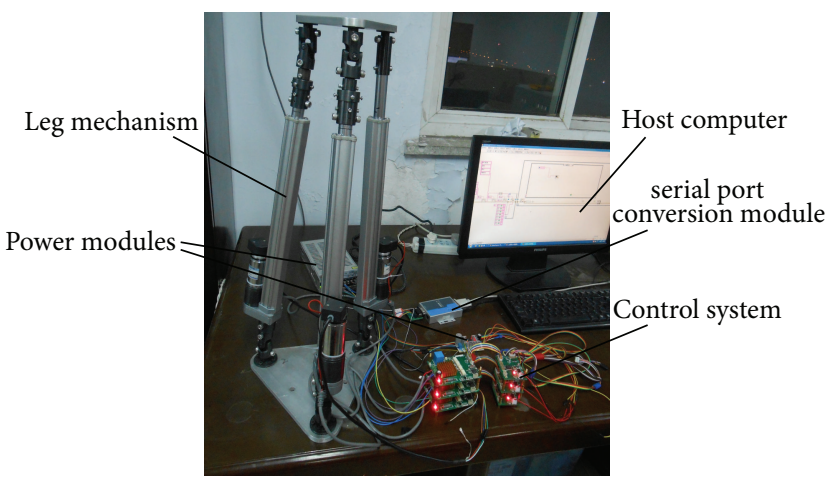

Figure 8: Hardware system of the leg mechanism.

control process is shown in Figure 10. The velocity control is firstly used between two discrete points, and then the position control is adopted when the distance between current position and the second discrete point is less than $\Delta q$. The velocity is controlled by using trapezoidal control mode and the acceleration and deceleration cycle could be set according to the requirement.

6.2. Experiment Research of the Leg Mechanism. To versify feasibility of the compound position control, experiment research is conducted in the optimal motion workspace which is obtained in Section 5.2. The point $E_{1}$ on the lower platform moves according to trajectory of the following equation:

$$
\begin{aligned}
& X_{E}=100 \cos \left(\frac{2 \pi}{5} t-\frac{\pi}{2}\right), \\
& Y_{E}=100+100 \sin \left(\frac{2 \pi}{5} t-\frac{\pi}{2}\right), \\
& Z_{E}=-675.4 .
\end{aligned}
$$

On basis of (37) and inverse kinematics of the leg mechanism, the position and velocity of the three branches could be computed by the simulation software, as shown in Figure 11.

According to the design of the leg mechanism, the length of branch one is in the range $591.4 \leq l_{11} \leq 891.4 \mathrm{~mm}$ and the velocity is in the range $0 \leq v_{1} \leq 80 \mathrm{~mm} / \mathrm{s}$; the length of branches two and three is in the range $574 \leq l_{1 i} \leq 874 \mathrm{~mm}$ and the velocity is in the range $0 \leq v_{i} \leq 80 \mathrm{~mm} / \mathrm{s}$. Observing Figures 11(a) and 11(b), we could find that the values of the three branches are in the motion range and the trend of their motion is correct.

The position and velocity of three branches are obtained by offsetting the simulation data, and then the results are saved as a binary file to load into the control program. The motion of the 2-UPS+UP PM for $5 \mathrm{~s}$ is controlled by the control program and the result of the movement is shown in Figure 12. The run time of the controller for each control cycle is $0.464 \mathrm{~ms}$, and a set of the movement data which consist of position data and velocity data is taken out at regular intervals with 256 control cycles. Therefore, in the process of the whole movement, a maximum of 42 sets of data could be saved. The curves are formed by using 42 sets of data, as shown in Figure 13.

Observing Figure 13, the following conclusions could be obtained.

(1) The real position trajectories of the leg mechanism are stable and fluctuation is not obvious. So using the compound position control strategy is suitable for the human-carrying walking chair robot.

(2) In some areas, the velocity of the branch is zero such as the starting, crossing zero, and the end, as shown in Figure 12(b). The cause of this result is that no data 


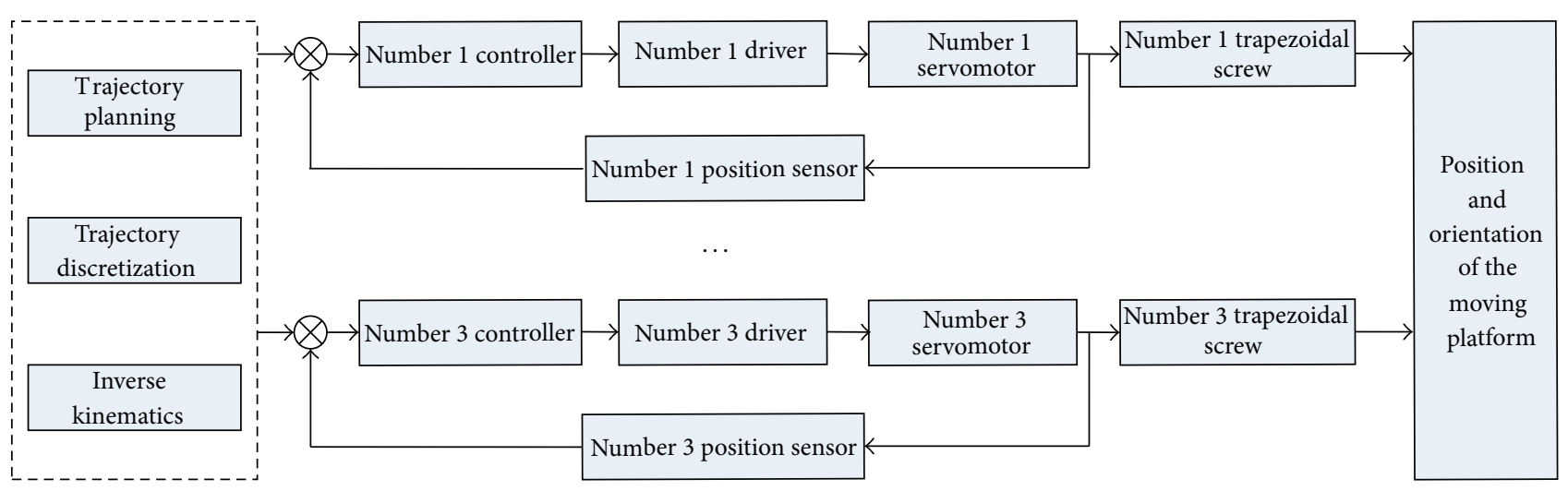

FIGURE 9: Control block diagram in the joint space.

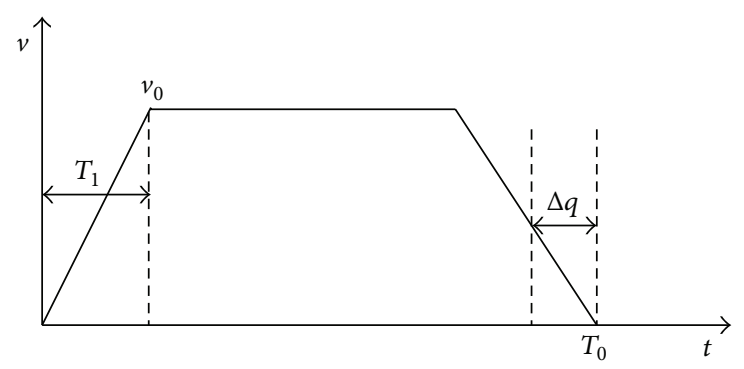

FIgURE 10: Compound position control.

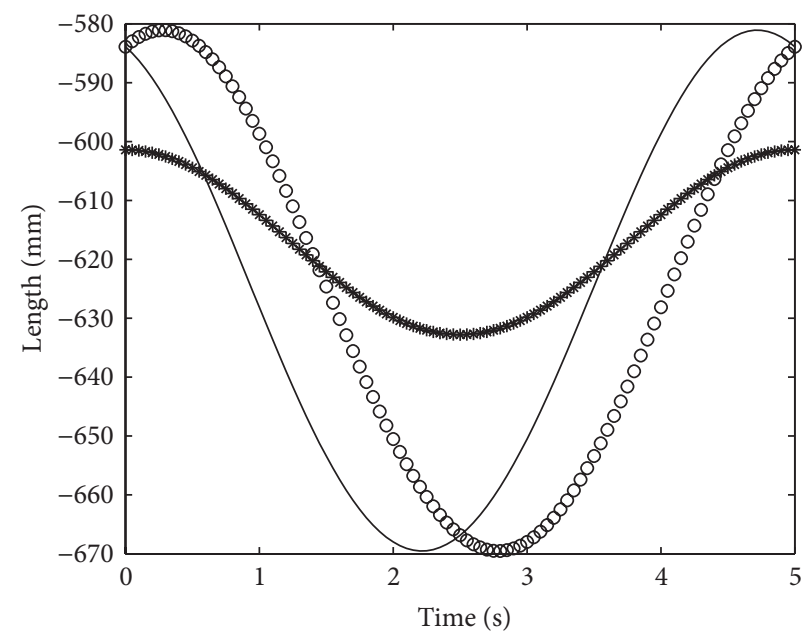

$\begin{array}{ll}* & l_{11} \\ & l_{12} \\ \circ & l_{13}\end{array}$

(a) The length change of three branches

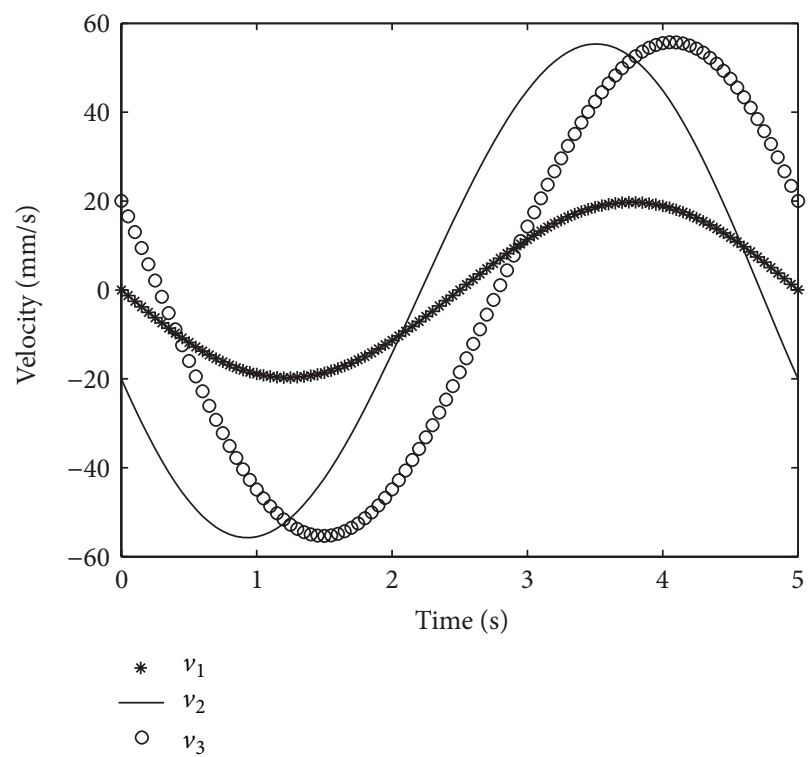

(b) Velocity change of three branches

FIGURE 11: Inverse kinematics analysis of the leg mechanism.

are returned from the controller when the velocity is smaller (about $v_{i} \leq 5 \mathrm{~mm} / \mathrm{s}$ ).

(3) The runtime of the leg mechanism is about $4.75 \mathrm{~s}(40 \times$ $256 \times 0.464 / 1000 \approx 4.75 \mathrm{~s}$ ). It is quite similar to the simulation time.

Based on the above experiment analysis, we could know that this controller is not suitable for the precise control requirements, but it is enough for the control system of the human-carrying walking chair robot because control accuracy is undemanding.

\section{Conclusions}

Conclusions are as follows. 


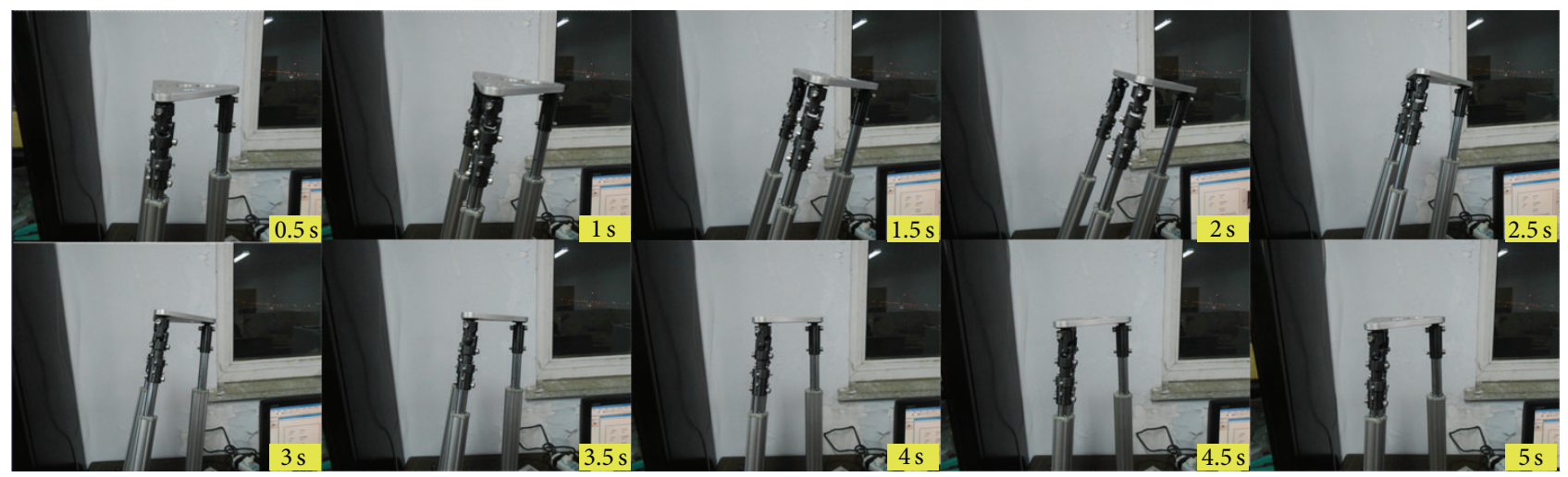

FIGURE 12: Movement of 2-UPS+UP PM for $5 \mathrm{sec}$.

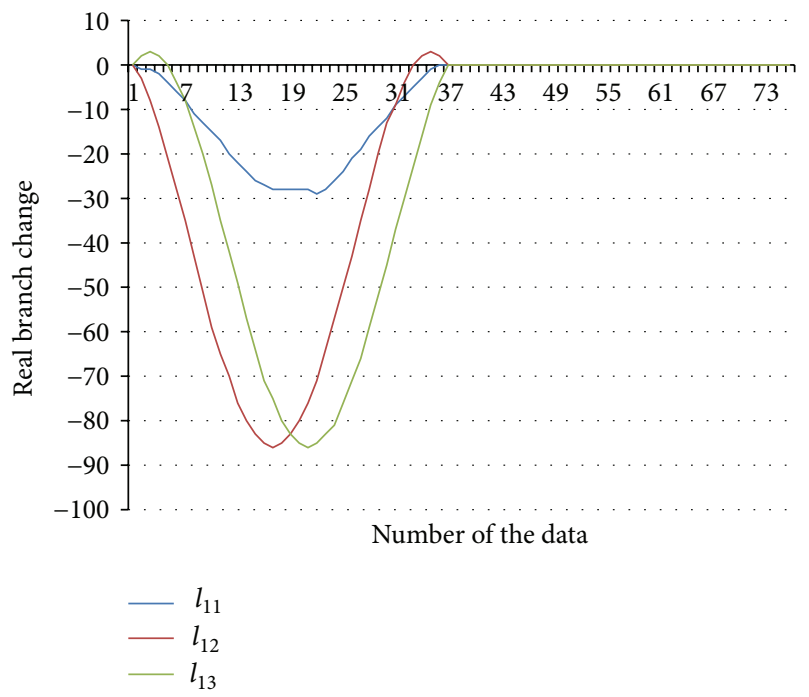

(a) The actual length change of three branches

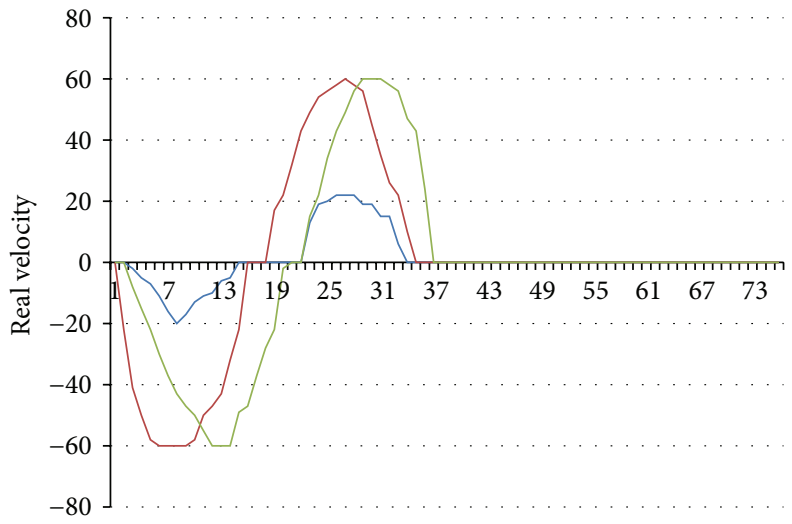

Number of the data

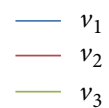

(b) The actual velocity of three branches

FIGURE 13: Real movement of three branches of the leg mechanism.

(1) Based on theory analysis (such as forward position analysis, inverse position analysis, and inverse velocity analysis), the structural constraint and condition number, and the optimal workspace of 2-UPS+UP $\mathrm{PM}$ are obtained.

(2) The control system of the leg mechanism for the quadruped human-carrying walking chair robot is designed, and the compound position control strategy is studied. Through experiment verification, it is found that the control strategy is not suitable for the precise control requirements, but it is enough for the control system of walking chair robot.

(3) In the experimental process, the real-time operation is conducted by host computer, but the operating system of host computer influences the time of sending and receiving data. So, in the future design of the control system of the whole walking chair robot, single chip computer is added to improve the realtime performance.

\section{Conflict of Interests}

The authors declare that there is no conflict of interests regarding the publication of this paper.

\section{Acknowledgments}

This work is supported by National Natural Science Foundation of China (no. 61075099), EU RABOT Project (FP7PEOPLE-2012-IRSES: no. 318902), and the High Level Talent Project in Hebei Province.

\section{References}

[1] W. Ping, X. M. Dun, and W. D. Chen, "The general overview of research on assistant robot," Robot Technique and Application, no. 1, pp. 31-34, 2009.

[2] J. Liu, X.-G. Zhao, and M. Tan, "Legged robots: a review," Robot, vol. 28, no. 1, pp. 81-88, 2006.

[3] J. H. Zhang, X. J. Zhang, M. L. Zhang, and F. H. Guo, "Eight wheel-legged mobile robot platform design and kinematic 
analysis," Chinese Journal of Machine Design, vol. 29, no. 8, pp. 35-39, 2012.

[4] C. Y. Zheng, Q. F. Zhao, P. S. Ma, H. Q. Zhang, and Z. Gou, "Mechanism design of a biped walking-chair robot," Robot, vol. 28, no. 3, pp. 297-302, 2006 (Chinese).

[5] K. Stefan, "On the anticipation of ethical conflicts between humans and robots in Japanese Mangas," International Review of Information Ethics, vol. 6, no. 12, pp. 63-68, 2006.

[6] J.-H. Kim, J.-Y. Kim, and J.-H. Oh, "Adaptive walking pattern generation and balance control of the passenger-carrying biped robot, HUBO FX-1, for variable passenger weights," Autonomous Robots, vol. 30, no. 4, pp. 427-443, 2011.

[7] S. Nakajima and E. Nakano, "Adaptive gait for large rough terrain of a leg-wheel robot (Fifth report: integrated gait)," Journal of Robotics and Mechatronics, vol. 21, no. 13, pp. 419-426, 2009.

[8] K. Yoneda, "Light weight quadruped with nine actuators," Journal of Robotics and Mechatronics, vol. 19, no. 2, pp. 160-165, 2007.

[9] J. Tang, Q. Zhao, and R. Yang, "Stability control for a walkingchair robot with human in the loop," International Journal of Advanced Robotic Systems, vol. 6, no. 2, pp. 115-120, 2009.

[10] Y. Sugahara, G. Carbone, K. Hashimoto, M. Ceccarelli, H. O. Lim, and A. Takanishi, "Experimental stiffness measurement of WL-16RII biped walking vehicle during walking operation," Journal of Robotics and Mechatronics, vol. 19, no. 3, pp. 272-280, 2007.

[11] Y. Rong and Z.-L. Jin, "Dynamic modeling of 3-DOF parallel mechanical leg and peak prediction of servo motor," Optics and Precision Engineering, vol. 20, no. 9, pp. 1974-1983, 2012.

[12] H. Wang, Z. Qi, Z. Hu, and Z. Huang, "Application of parallel leg mechanisms in quadruped/biped reconfigurable walking robot," Chinese Journal of Mechanical Engineering, vol. 45, no. 8, pp. 24-30, 2009.

[13] L. W. Tsai and S. Joshi, "Kinematics and optimization of a spatial 3-UPU parallel manipulator," Journal of Mechanical Design, vol. 122, no. 4, pp. 439-446, 2000.

[14] Z. H. Guo, S. S. Sun, X. Q. Hao, G. D. Niu, and L. S. Li, "Position analysis and simulation of 3-PUU translation parallel manipulator," China Mechanical Engineering, vol. 17, no. 17, pp. 1787-1789, 2006.

[15] Q. Li, Z. Chen, Q. Chen, C. Wu, and Z. Huang, "Structural condition for [PP]S parallel mechanism without parasitic motion," Journal of Mechanical Engineering, vol. 46, no. 15, pp. 31-35, 2010.

[16] A. Kuma and K. J. Waldron, "The workspace of a mechanical mechanism," ASME Journal of Mechanical Design, vol. 103, pp. 665-672, 1981.

[17] T. Huang, J. Wang, C. M. Gosselin, and D. J. Whitehouse, "Kinematic synthesis of hexapods with specified orientation capability and well-conditioned dexterity," Journal of Manufacturing Processes, vol. 2, no. 1, pp. 36-47, 2000.

[18] H. Yu, L.-N. Sun, P.-K. Liu, and H.-G. Cai, "Study on the workspace and parameter of novel 6-HTRT parallel robot," Robot, vol. 24, no. 4, pp. 293-299, 2002.

[19] Y. Cao, Y. Zhang, and Y. Ma, "Workspace analysis and parameter optimization of 6-RSS parallel mechanism," Chinese Journal of Mechanical Engineering, vol. 44, no. 1, pp. 19-24, 2008.

[20] X. J. Liu, L. J. Zhang, and F. Gao, "Geometrical determination of workspace for 6-dof parallel manipulators based on AutoCAD platform," Robot, vol. 22, no. 6, pp. 457-465, 2000.
[21] Y. Lu, P. Wang, Z. Hou, B. Hu, C. Sui, and J. Han, "Kinetostatic analysis of a novel 6-DoF 3UPS parallel manipulator with multifingers," Mechanism and Machine Theory, vol. 78, pp. 36-50, 2014.

[22] I. A. Bonev and C. M. Gosselin, "Geometric algorithms for the computation of the constant-orientation workspace and singularity surfaces of a special 6-RUS parallel manipulator," in Proceedings of the ASME International Conference on Design Engineering Technical, Computers and Information in Engineering, vol. 10, pp. 1-10, ASME, Montreal, Canada, 2002.

[23] G. Cheng, J. Yu, X. Yuan, Y. Pang, and P. Xu, "Study on workspace optimization of 3SPS+1PS parallel hip joint simulator," Journal of Mechanical Engineering, vol. 49, no. 23, pp. 88-95, 2013.

[24] Z. Huang, Y. S. Zhao, and T. S. Zhao, Advanced Spatial Mechanism, Higher Education Press, Beijing, China, 2006. 

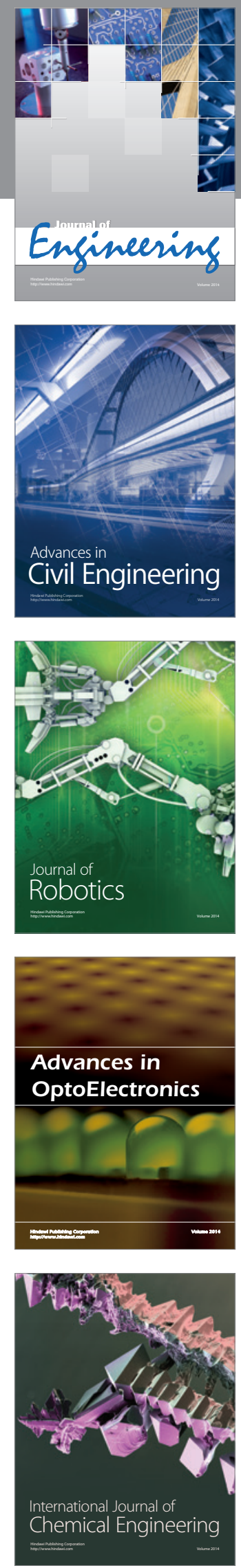

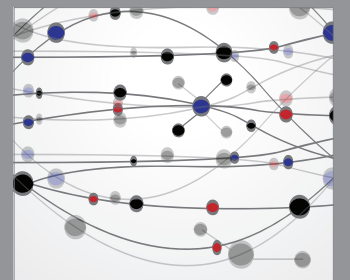

The Scientific World Journal
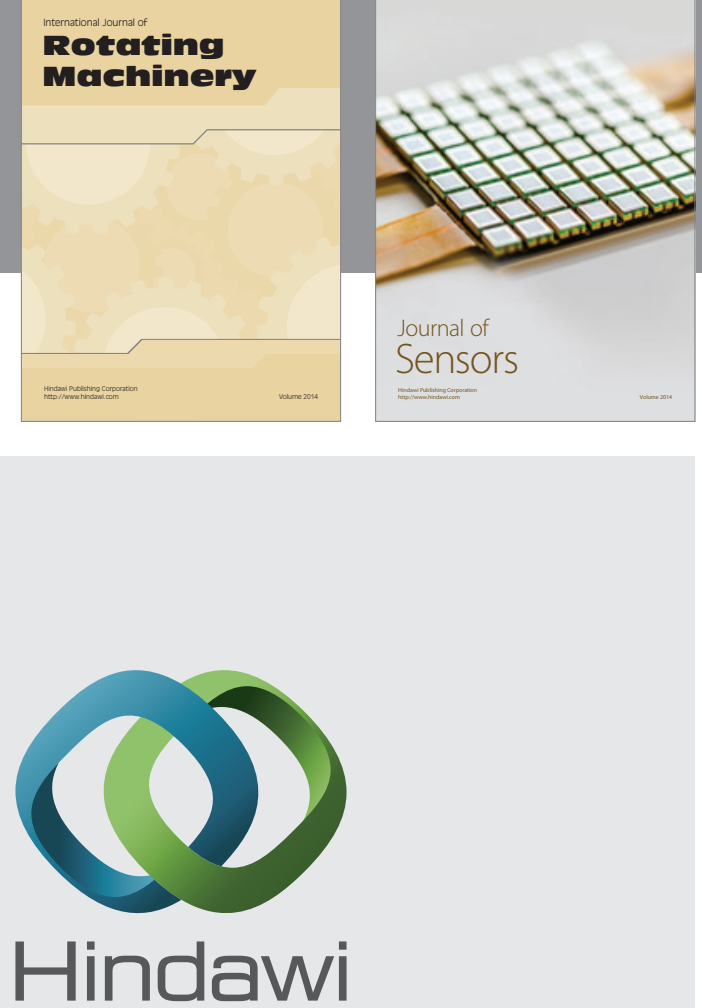

Submit your manuscripts at http://www.hindawi.com
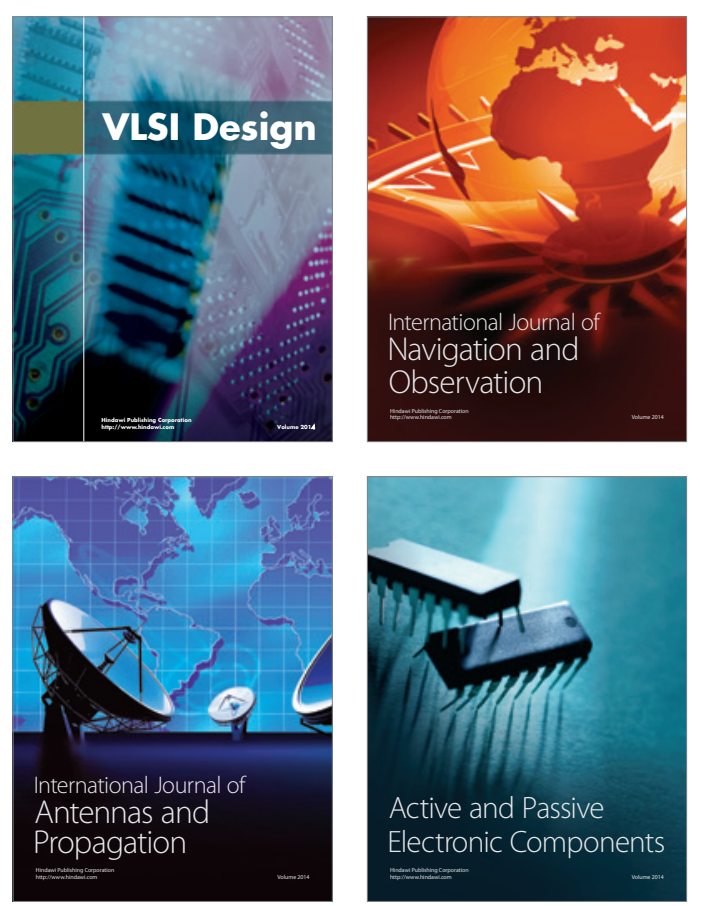
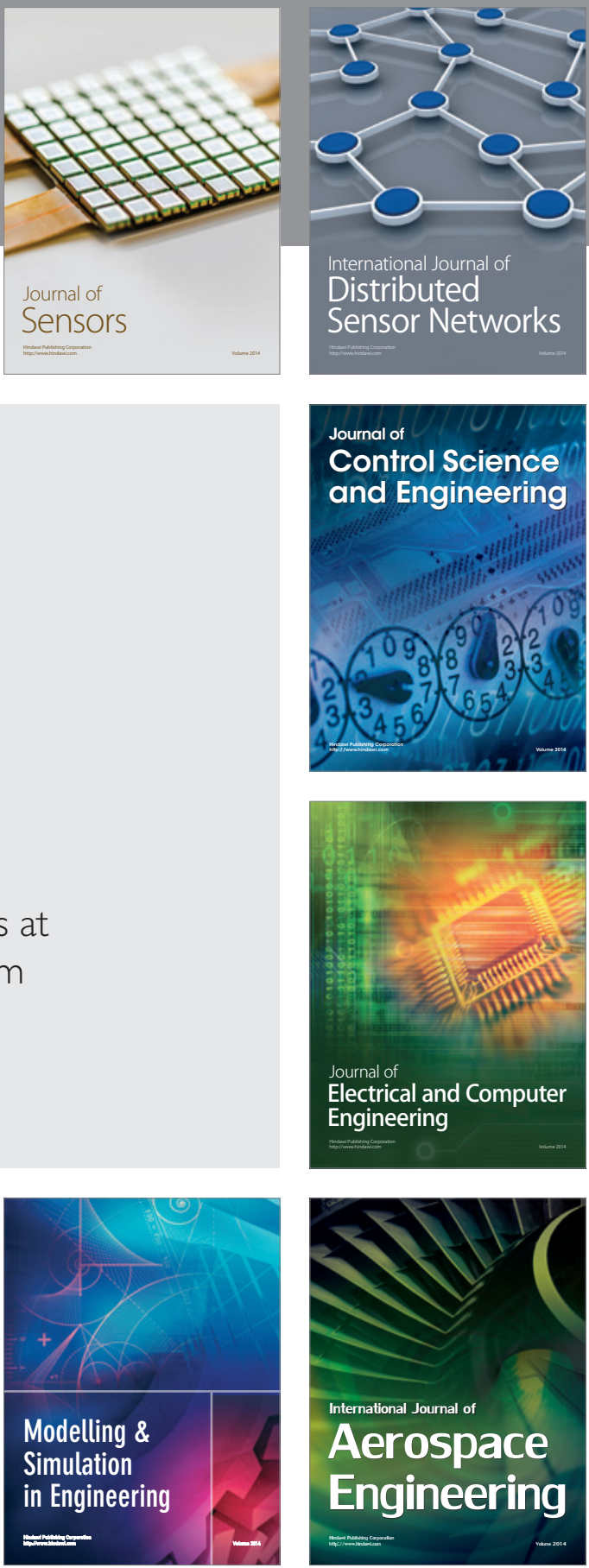

Journal of

Control Science

and Engineering
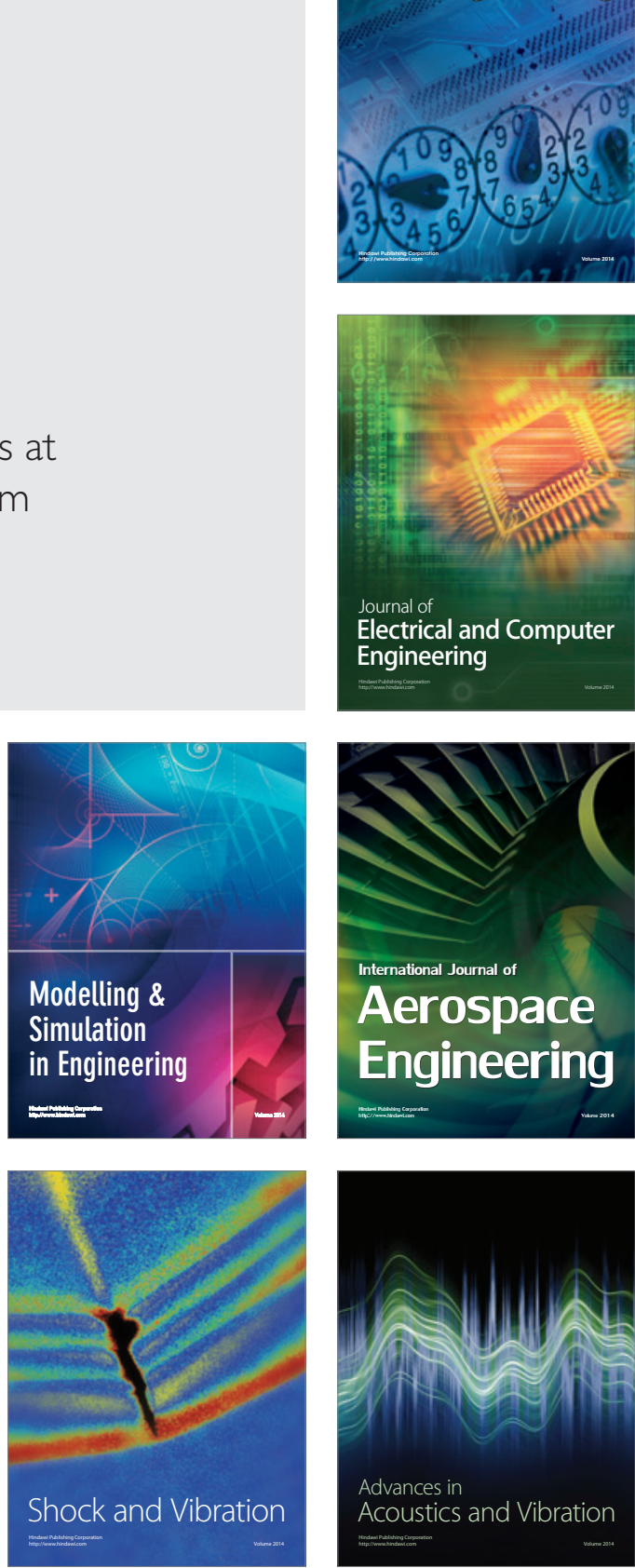